\title{
Analysis of Characteristic of Free Particles: Relativistic Concept
}

\author{
Emmanuel I. Ugwu', Daniel Ugadu Onah ${ }^{1}$, D. Oboma ${ }^{1}$, V. O. C. Eke ${ }^{2}$ \\ ${ }^{1}$ Departments of Industrial Physics, Ebonyi State University, Abakaliki, Nigeria \\ ${ }^{2}$ Departments of Computer Science, Ebonyi State University, Abakaliki, Nigeria \\ Email: ugwuei@yahoo.com
}

Received July 19, 2012; revised August 30, 2012; accepted September 10, 2012

\begin{abstract}
A linear Hamiltonian in spatial derivative that satisfies Klein-Gordon equation was used starting from energy momentum relation for free particle was solved in agreement with the matrices $\hat{\alpha}$ and $\hat{\beta}$ bearing in mind their suitability in terms of anticommutation relations in parallel with the definition of algebraic matrices whose hermicity is fulfilled by $\hat{\alpha}_{i} \hat{\alpha}^{\dagger}=\hat{\alpha}_{i}$ and $\hat{\beta}^{\dagger}=\hat{\beta}$ and in turn linked up to explicit representation of the Dirac matrices. The wave packets of plane Dirac wave obtained as a superposition of plane wave yielding a localized wave function was normalized considering only positive energy of plane wave in which the expectation value with respect to the wave packet resulted from $\left\langle\frac{c^{2} p}{E}\right\rangle_{+}=\left\langle\left(v_{g r}\right)\right\rangle$ was found to agree with the Ehrenfest theorem in relation to Schrodinger theorem as it relates to true velocity of single particle. A comparison was made between the classical concept with Heisenberg representation from where the combined effect of the positive and negative energy components was considered.
\end{abstract}

Keywords: Hamiltonian; Klein-Gordon Equation; Wave Function; Dirac Matrices Wave Packet; Heisenberg Representation; Superposition; Energy; Group Velocity; Frequency

\section{Introduction}

The of the wave particle plays important role in understanding of the behavourial description of particle as regards the electronic structure, energy spectrum and wave characteristics [1]. With regard to this, many researchers probe into this using classical approach which was of course found to be insufficient in giving complete description particle. However this is because relativistic effect which is of most important is in description of any physical system is excluded. Indeed relativistic effect plays a crucial role the description of electronic structure of not only small particle but also heavy particles [1]. Sequel to the evolution of Dirac theory that led to formulation Dirac equation, Dirac's relativistic frame work where one electron spinorial solution is a two vector whose component are wave function has been used but was found incomplete in giving description of particle [2-4]. A survey of the current available analytical solution using relativistic one electron atoms has been carried out.It has been made that reasonable description can only be given by making use of quantum electrodynamics [5].

In this work we intend to analyze the wave character- istics using relativistic approach by bringing together momentum relation, vectorial relation and Dirac wave in conjunction with Heisenberg representation observe in a real sense the feasibility of existence of single particle.

\section{Analytical Procedures}

The analytical procedure is taken in three aspects via momentum relation and matrices of free particle, Dirac wave and Heisenberg representation.

\subsection{Momentum Relation and Matrices of Free Particle}

We start with relativistic covariant wave equation of Schrodinger form is written as

$$
-\hbar \frac{\partial \psi}{\partial t}=\hat{H} \psi
$$

for free particle $[5,6]$.

This is a linear time derivative. Now we construct a Hamiltonian that is also linear in the spatial derivative that is of the form 
$\hat{H}_{f} \psi \equiv i \hbar \frac{\partial \psi}{\partial t}\left[\frac{\hbar c}{i}\left(\hat{\alpha}_{1} \frac{\partial}{\partial x^{1}}+\hat{\alpha}_{2} \frac{\partial}{\partial y^{2}}+\hat{\alpha}_{3} \frac{\partial}{\partial z^{3}}\right)+\hat{\beta} m_{o} c^{2}\right] \psi$

Of which if every single component, $\psi_{\alpha}$ of the wave function has to satisfy Klein-Gordon equation [4,5,7] starting from energy momentum relation for free particle

$$
E^{2}=p^{2} c^{2}+m_{0}^{2} c^{4}
$$

In terms of Equation (1), we write

$$
\hbar^{2} \frac{\partial^{2} \psi_{\alpha}}{\partial t^{2}}=\left[-\hbar^{2} c^{2} \nabla^{2}+m_{o}^{2} c^{4}\right] \psi_{\alpha}
$$

This can be iterated to give

$$
\begin{aligned}
-\hbar^{2} \frac{\partial^{2} \psi}{\partial t^{2}}= & {\left[\hbar^{2} c^{2 \sum_{i, j=1}^{3} \frac{\hat{\alpha}_{i} \hat{\alpha}_{j}-\hat{\alpha}_{j} \hat{\alpha}_{i}}{2}} \frac{\partial^{2}}{\partial x^{i} \partial x^{j}}\right.} \\
& \left.+\frac{\hbar m_{o} c^{2}}{i} \sum_{i=1, j}^{2}\left(\hat{\alpha}_{i} \hat{\beta}+\hat{\beta} \hat{\alpha}_{j}\right) \frac{\partial}{\partial x^{i}}+\hat{\beta}^{2} m_{o} c^{4}\right] \psi
\end{aligned}
$$

This expression has to agree with the following requirements for the matrices $\hat{\alpha}$ and $\hat{\beta}$ to be suitable for our application. These are

$$
\begin{gathered}
\hat{\alpha}_{i} \hat{\alpha}_{j}=\hat{\alpha}_{j} \hat{\alpha}_{i}=2 \delta_{i j} \| \\
\hat{\alpha}_{i} \hat{\beta}=\hat{\beta} \hat{\alpha}_{i}=0 \\
\hat{\alpha}_{i}^{2}=\hat{\beta}^{2}=\|
\end{gathered}
$$

These anticommutation relations defines the algebraic matrices whose hermicity is fulfilled if

$$
\hat{\alpha}_{i} \hat{\alpha}_{i}^{\dagger}=\hat{\alpha}_{i} \text { and } \hat{\beta}^{\dagger}=\hat{\beta}
$$

with $\hat{\alpha}_{i}^{2}=1$ and $\hat{\beta}^{2}=1$. This implies that the eigenvalues have the values \pm 1 . One of the special features exhibited by the eigenvalue is the fact that they are independent of special representation and can be shown as diagonal representation of the form with the eigenvalues

$$
A_{1} \cdots A_{N}
$$

were

$$
\hat{\alpha}_{i}=-\hat{\alpha} \|=\left(\begin{array}{cccc}
1 & 0 & 0 & \ldots \\
0 & 1 & 0 & \ldots \\
0 & 0 & 1 & \ldots \\
\vdots & \vdots & \vdots & \ddots
\end{array}\right)
$$

From the anticommutation relation of Equation (6) it is generalize that trace, i.e. the sum of the diagonal elements of the matrices of each $\hat{\alpha}$ and $\hat{\beta}$ has to be zero representation.

i.e.

$$
\hat{\alpha}_{i}=-\hat{\beta} \hat{\alpha} \beta
$$

As

$$
\operatorname{tr} \hat{A} \hat{\beta}=\operatorname{tr} \hat{\beta} \hat{A}
$$

which invariably indicates that

$$
\operatorname{tr} \hat{\alpha}_{i} \equiv \operatorname{tr} \hat{\beta} \hat{\alpha}_{i} \hat{\beta}=-\operatorname{tr} \hat{\alpha}_{i}
$$

Indicating that

$$
\operatorname{tr} \hat{\alpha}_{i}=0
$$

This agrees with the practice that the trace of a matrix $[8,9]$ is always equal to the sum of its eigenvalues. i.e.

$$
\begin{aligned}
& \operatorname{tr}\left(\begin{array}{cccc}
A_{1} & 0 & 0 & \cdots \\
0 & A_{2} & 0 & \ldots \\
\vdots & \ddots & A_{N} & \ldots
\end{array}\right) \\
& =\sum_{K=1}^{N} A_{K}=\operatorname{tr} \hat{u} \hat{\alpha} \hat{u}^{-1} \\
& \Rightarrow \operatorname{tr} \alpha_{i} \hat{u} \hat{u}^{-1}=\operatorname{tr} \alpha_{i}
\end{aligned}
$$

where

$$
K A_{K}^{2}=1=A_{K}= \pm 1
$$

Since the explicit representation of the Dirac matrices are

$$
\begin{aligned}
& \hat{\alpha}_{i}=\left(\begin{array}{cc}
0 & \hat{\alpha}_{i} \\
\hat{\alpha} & 0
\end{array}\right) \\
& \hat{\beta}=\left(\begin{array}{cc}
11 & 0 \\
0 & -11
\end{array}\right)
\end{aligned}
$$

With $\hat{\alpha}_{i}$ being Pauli's matrices and 11 unit matrix $\hat{\alpha}_{i}$ and $\hat{\beta}$ can be written especially as shown below.

$$
\begin{aligned}
\hat{\alpha}_{1} & =\left(\begin{array}{llll}
0 & 0 & 0 & 1 \\
0 & 0 & 1 & 0 \\
0 & 1 & 0 & 0 \\
1 & 0 & 0 & 0
\end{array}\right) \\
\hat{\alpha}_{2} & =\left(\begin{array}{cccc}
0 & 0 & 0 & -i \\
0 & 0 & i & 0 \\
0 & -i & 0 & 0 \\
i & 0 & 0 & 0
\end{array}\right) \\
\hat{\alpha}_{3} & =\left(\begin{array}{cccc}
0 & 0 & 1 & 0 \\
0 & 0 & 0 & -1 \\
1 & 0 & 0 & 0 \\
0 & -1 & 0 & 0
\end{array}\right) \\
\hat{\beta} & =\left(\begin{array}{cccc}
1 & 0 & 0 & 0 \\
0 & 0 & 0 & -1 \\
0 & 0 & -1 & 0 \\
0 & 0 & -0 & -1
\end{array}\right)
\end{aligned}
$$




\subsection{Dirac Wave}

By considering only a free particle we set

$$
\hat{p} \psi=0
$$

This makes Equation (2) to become

$$
\hbar \frac{\partial \psi}{\partial t}=\hat{\beta} M_{0} C^{2} \psi^{\prime}
$$

of which we can write four solutions of wave function thus

$$
\begin{aligned}
& \psi^{(1)}=\left(\begin{array}{l}
1 \\
0 \\
0 \\
0
\end{array}\right) \exp \left[-i\left(M_{0} C^{2} / \hbar\right) t\right] \\
& \psi^{(2)}=\left(\begin{array}{l}
0 \\
1 \\
0 \\
0
\end{array}\right) \exp \left[-i\left(M_{0} C^{2} / \hbar\right) t\right] \\
& \psi^{(3)}=\left(\begin{array}{l}
0 \\
0 \\
1 \\
0
\end{array}\right) \exp \left[+i\left(M_{0} C^{2} / \hbar\right) t\right] \\
& \psi^{(4)}=\left(\begin{array}{l}
0 \\
0 \\
0 \\
1
\end{array}\right) \exp \left[+i\left(M_{0} C^{2} / \hbar\right) t\right]
\end{aligned}
$$

of which the first two correspond to positive energy value while the last two are negative energy values. In addition to this unique properties exhibited by Dirac equation, it also depicts covariance properties. This on the other hand explains that the solution appears to possess the correct behaviour in the non relativistic limit in agreement with the considered the wave packet. The wave packets of plane Dirac waves are superpositions of plane wave yield localized wave functions in space and time since Dirac equation is a linear wave equation that marches a wave packet of a plane wave with positive energy $[3,7]$. They are of the form.

$$
\psi^{(5)}\left(x_{1} t\right)=\int \frac{\mathrm{d}^{3} p}{\sqrt{2 \pi \hbar^{3}}} \sqrt{\frac{M_{0} C^{2}}{E} \sum_{\neq s} b(p, s) u(p, s)} e^{-i\left(p_{0}-p_{0}^{1}\right) x_{0} / \hbar}
$$

Here the amplitude $b(p, s)$ determines the admixture of the plane wave $u(p, s) e^{-} \psi_{i} x^{\prime \prime} / h$ to the wave packet and the plus indicates that a superposition of only positive energy plane wave is considered.

This is normalized as

$$
\int \psi^{(+)+}(x, t) \psi^{(+)}(\alpha, t) \mathrm{d}^{3} x=1
$$

with this normalization [7], the expectation value with respect to the wave packet of positive energy is written is written as

$$
J_{i}^{+}=\int \mathrm{d}^{3} p \frac{p_{i} c^{2}}{E} \sum_{ \pm S}|b(p, s)|=\left\langle\frac{c^{2} p_{i}}{E}\right\rangle_{+}=\left\langle\left(V_{g r}\right)_{i}\right\rangle_{+}
$$

Similarly the mean current of an arbitrary wave packet of plane wave of positive energy from non relativistic concept is equal to the expectation value the classical group velocity,

$$
V_{g r}=\frac{c^{2} p}{E}
$$

This corresponds to the Ehrenfest theorem on relation to Schrödinger theory and invariably agrees with the true velocity of single particle that constitutes. $+c^{2} \hat{p} / E_{p}$ and $-\frac{c^{2} \hat{p}}{E_{p}}$ for free solutions of the Dirac equation. This assertion agrees with the classical picture.

\subsection{Heisenberg Representation}

On the other hand, for waves with the same characteristics $[7,10,11]$, the result exhibits paradoxical nature as their velocity is directed against their momentum which explains the fact that those particles with negative energy apparently appears to behave as if they have a negative mass. Further clarification can be made using the equation of motion in the Heisenberg representation.

$$
\frac{\mathrm{d} \hat{x}}{\mathrm{~d} t}=\frac{1}{i h \hbar}\left[\hat{x}, H_{f}\right]=c \hat{\alpha}
$$

This can be solved by comparing it with the classical equation of motion of a rapidly oscillating motion

$$
\left\langle\left|i \propto \hat{\propto}(0)-\frac{c \hat{p}}{H_{f}} \exp \left(-2 i H_{f} t / \hbar\right)\right|\right\rangle
$$

Whose amplitude and frequency of these additional oscillations are of the other $\hbar / 2 m_{0} c$ and $\frac{\hbar}{2 m_{0} c^{2}}$ respectively. This oscillating motion as mentioned already is known as Zitterbewegung which vanishes if wave packets with exclusively positive or negative energy are considered. This implies that interference effect resulting from the combined effect of the positive and negative energy components of a wave packet and invariably demonstrates that in real sense a single particle theory is not absolutely possible in practice but can only be approximately obtained if the associated wave packet is restricted to one energy range. The most interesting as- 
pect observed in this analysis is that the true velocity of single particle appears to constitute both the negative and positive terms of energy which brings about the idea that a particles with negative energy apparently tends to have negative mass as their velocity is directed against their momentum.

General observation shows that the mean current of an arbitrary wave packet plane energy from non relativist concept agrees with the expectation value of the classical group velocity $\left[V_{g r}=\frac{c^{2} p}{E}\right]$ which corresponds to the Ehrenfest theorem in relation to Schrodinger theory [3] further clarification during the analysis using Heisenberg representation brings about frequency of additional oscillations of the order

$$
\hbar / 2 m_{0} c \text { and } \frac{\hbar^{2}}{2 m_{0} c^{2}}
$$

\section{Discussion}

In this paper we have analytically studied the fundamental wave characteristics of free particle based on equation constructed from relativistically covariant wave equation of Schrodinger equation that satisfies Klein-Gordon equation. The expression for anticommutation relations that defined the algebraic matrices with special representation showing the diagonal matrices $A$, to $A_{N}$ in conjunction with the explicit representation of the Dirac matrices are seen in Equations (14a) and (14b) with their explicit value presented in Equations (15)-(18). The wave func- tion relating to wave packet of the plane wave associated with any particle being described using Dirac equation is presented in Equations (21)-(24) as wave packets. The packet explains the fact that Dirac waves are superposition plane waves and yields localized wave functions in space and time in which when considered with only positive energy of a particle is compactly written as in Equation (35) and normalized as in Equation (26). The normalization enabled us to write the expectation value with respect to the wave packet as seen in Equation (27) is known as Zitterbewegung that vanishes if the wave packet associated exclusively to both positive and nega- tive energy. This implies that Zitterbewegung is as a result of the combined effect of negative and positive energy components a situation that confirmed the fact that in a real sense of it, the idea of a single particle is feasible only when it is considered with restriction to one energy range.

\section{REFERENCES}

[1] A. L. A. Fonseca, D. L. Nascimento, F. F. Monteiro and M. A. Amato, "A Variational Approach for Numerically Solving the Two-Component Radial Dirac Equation for One-Particle Systems," Journal of Modern Physics, Vol. No. 4, 2012, pp. 350-354.

[2] R. Franke, "Numerical Study of the iterated solution of one electron Dirac Equation based on 'Dirac Perturbation theory'," Chemical Physics Letters, Vol. 264, No. 5, 1997 pp. 495-501. doi:10.1016/S0009-2614(96)01361-9

[3] S. McConnel, S. Fritzsch and A. Surzhykoy, "Dirac: A New Version of Computer Algebra Tools for Studying the Properties and Behaviour of Hydrogen-Like Ions," Computer Physics Communication, Vol. 181, No. 3, 2010, pp. 711-713.

[4] A. Surzhykoy, P. Koval and S. Fritzsch, "Algebraic Tools for Dealing with Atomc Shell Model. 1. Wavefunctions and Itegrals for Hydrogen-Like Ions," Computer Physics Communication, Vol. 165, No. 2, 2005, pp. 139-156. doi:10.1016/j.cpc.2004.09.004

[5] A. Zee, "Quantum Field Theory in Nutshell," Princeton University Press, Princeton, 2010.

[6] P. W. Atkins, "Molecular Quantum Mechanics," Oxford University Press, Oxford, 1983.

[7] R. A. C. Dirac, "The Lagrangian in Quantum Mechanics," Physikalisch Zeitchrift der Sowjetunion, Vol. 3, 1933, pp. 62-72.

[8] R. Ballan and J. Zinn-Justin, "Methods in Field Theory," North Holland Publishing, Amsterdam and World Scientific, Singapore City, 1981.

[9] P. A. M Dirac, "Principle of Quantum Mechanics," Oxford University Press, Oxford, 1935.

[10] E. G. Milewski, "Vector Analysis Problem Solver," Research and Education Association, New York, 1987.

[11] L. H. Ryder, "Quantum Field Theory," Cambridge University Press Cambridge, 1996. 